\title{
Birth outcomes associated with cannabis use before and during pregnancy
}

\author{
Mohammad R. Hayatbakhsh 1,2, Vicki J. Flenady², Kristen S. Gibbons², Ann M. Kingsbury², Elizabeth Hurrion², \\ Abdullah A. Mamun' and Jake M. Najman'
}

INTRODUCTION: This study aimed to examine the association between cannabis use before and during pregnancy and birth outcomes.

RESULTS: Overall, $26.3 \%$ of women reported previous use of cannabis and $2.6 \%$ reported current use. Multivariate analysis, controlling for potential confounders, including tobacco smoking, alcohol consumption, and use of other illicit drugs, showed that cannabis use in pregnancy was associated with low birth weight (odds ratio $(O R)=1.7 ; 95 \%$ confidence interval (Cl): 1.3-2.2), preterm labor $(\mathrm{OR}=1.5 ; 95 \% \mathrm{Cl}$ : 1.1-1.9), small for gestational age $(\mathrm{OR}=2.2 ; 95 \% \mathrm{Cl}: 1.8-2.7)$, and admission to the neonatal intensive care unit $(\mathrm{OR}=2.0 ; 95 \% \mathrm{Cl}: 1.7-2.4)$.

DISCUSSION: The results of this study show that the use of cannabis in pregnancy is associated with increased risk of adverse birth outcomes. Prevention programs that address cannabis use during pregnancy are needed.

METHODS: Data were from women birthing at the Mater Mothers' Hospital in Brisbane, Australia, over a 7-y period (2000-2006). Women were interviewed in the initial antenatal visit about their use of cannabis and other substances. Records for 24,874 women who provided information about cannabis use, and for whom birth outcomes data were available, were included in the analysis.

D espite increasing public health concerns, cannabis remains the most commonly used illicit drug among women of childbearing age in developed countries, including the United States and Australia (1,2). In a 2007 national survey, 30.0\% of Australian women aged 14 y or older had used cannabis at some time in their lives. Of the same Australian women, 6.6\% reported they had used cannabis in the past 12 mo, with a higher proportion reported by women of childbearing ages (3). It is suggested that the active ingredient of cannabis, tetrahydrocannabinol, crosses the placental barrier and directly affects the fetus. Persistent use of cannabis may result in decreased uteroplacental perfusion and intrauterine fetal growth restriction (4). Previous research linking antenatal cannabis use and adverse pregnancy outcomes has been inconclusive (5). A number of studies have reported some adverse effects, including low birth weight (6-9), preterm birth $(7,8,10)$, small for gestational age (SGA) (7), and small birth length $(11,12)$. However, other studies have disputed these findings $(10,12-18)$.

Although the volume of literature on the association between cannabis use in pregnancy and birth outcomes has been growing, there remains concern about the limitations of previous research. Limitations include small or highly selected samples $(8,12)$; lack of prospectively collected measures of cannabis use; and lack of control for potential confounders, e.g., socioeconomic status and maternal health, and also use of other substances (cigarettes, alcohol, and other drugs) (7). Cannabis users differ from nonusers in a range of ways and any observed association with ever using cannabis may be indicative of residual or uncontrolled confounding. Distinguishing between ever cannabis use and use in pregnancy and birth outcomes is of particular importance. As most cannabis users also use other substances, such as tobacco and alcohol, it is difficult to identify the specific effects of cannabis on the fetus (4). This study aims to examine the association between birth outcomes and use of cannabis prior to and during pregnancy, independent of potential confounding factors.

\section{RESULTS}

Between 2000 and 2006, 25,073 women presented to Mater Mothers' Hospital (MMH) for antenatal care. Of those, 24,874, for whom data were available on cannabis use before and during pregnancy, gave birth to live babies. They had mean age of 28.4 y (SD $=5.8$ y). Overall, $26.3 \%$ of women reported ever use of cannabis, 9.5\% had used cannabis regularly before, and $2.6 \%$ were using cannabis during pregnancy. Some $0.7 \%(n=161)$ reported use of other illicit drugs (heroin, amphetamines, ecstasy, or hallucinogens), of whom 63 admitted use of cannabis during pregnancy. Another 0.8\% (193) reported use of prescription methadone during pregnancy, of whom 47 were cannabis users. Table 1 shows the prevalence of cannabis use before and during pregnancy according to the women's sociodemographic characteristics. Cannabis use was more common among women who were younger, had lower levels of education, and were single across all categories of use, i.e., lifetime, ever regular, and use in pregnancy. Indigenous women and those who were nulliparous reported higher rates of cannabis ever

\footnotetext{
'School of Population Health, The University of Queensland, Brisbane, Australia; '2Mater Medical Research Institute, Mater Mothers' Hospital, Brisbane, Australia.
} Correspondence: Mohammad R. Hayatbakhsh (m.hayatbakhsh@uq.edu.au) 
Table 1. Univariate analysis of cannabis use by maternal characteristics, Mater Mothers' Hospital, 2000-2006

\begin{tabular}{|c|c|c|c|c|}
\hline \multirow[b]{3}{*}{ Characteristic } & \multirow[b]{3}{*}{$N$} & \multicolumn{3}{|c|}{ Cannabis use } \\
\hline & & Lifetime & $\begin{array}{l}\text { Ever } \\
\text { regular }\end{array}$ & $\begin{array}{c}\text { During } \\
\text { pregnancy }\end{array}$ \\
\hline & & $N(\%)$ & $N(\%)$ & $N(\%)$ \\
\hline Age & $P^{a}$ & $<0.001$ & $<0.001$ & $<0.001$ \\
\hline$<20 y$ & 2,342 & $1,059(45.2)$ & $480(20.5)$ & $146(6.2)$ \\
\hline $20-35 y$ & 19,597 & $4,974(25.4)$ & $1,734(8.9)$ & $451(2.3)$ \\
\hline$>35 y$ & 2,919 & $490(16.8)$ & $157(5.4)$ & $39(1.3)$ \\
\hline Highest education & $P^{a}$ & $<0.001$ & $<0.001$ & $<0.001$ \\
\hline Tertiary & 7,074 & $1,496(21.2)$ & $380(5.4)$ & $53(0.8)$ \\
\hline $\begin{array}{l}\text { Completed high } \\
\text { school }\end{array}$ & 8,688 & $2,340(26.9)$ & $763(8.8)$ & $161(1.9)$ \\
\hline $\begin{array}{l}\text { Incomplete high } \\
\text { school }\end{array}$ & 7,456 & $2,514(33.7)$ & $1,127(15.1)$ & $378(5.1)$ \\
\hline Unknown & 1,158 & $119(10.3)$ & $65(5.6)$ & $29(2.5)$ \\
\hline Marital status & $P^{a}$ & $<0.001$ & $<0.001$ & $<0.001$ \\
\hline $\begin{array}{l}\text { Married/living } \\
\text { together }\end{array}$ & 20,865 & $4,917(23.6)$ & $1,624(7.8)$ & $393(1.9)$ \\
\hline Single & 3,468 & $1,475(42.5)$ & $694(20.0)$ & $222(6.4)$ \\
\hline $\mathrm{S} / \mathrm{D} / \mathrm{W}$ & 541 & $139(25.7)$ & $56(10.4)$ & $22(4.1)$ \\
\hline Parity & $P^{a}$ & $<0.001$ & $<0.001$ & $<0.05$ \\
\hline Zero & 8,597 & $2,903(33.8)$ & $1,064(12.4)$ & $226(2.6)$ \\
\hline One & 8,723 & $2,144(24.6)$ & 735 (8.4) & $195(2.2)$ \\
\hline Two & 4,265 & $873(20.5)$ & $324(7.6)$ & $110(2.6)$ \\
\hline Three or more & 3,283 & $607(18.5)$ & $248(7.6)$ & $105(3.2)$ \\
\hline Ethnicity & $P^{a}$ & $<0.001$ & $<0.001$ & $<0.001$ \\
\hline Caucasian & 18,725 & $5,706(30.5)$ & $2,063(11.0)$ & $540(2.9)$ \\
\hline Indigenous & 930 & $382(41.1)$ & $175(18.8)$ & $71(7.6)$ \\
\hline Asian & 2,879 & $116(4.0)$ & $31(1.1)$ & $5(0.2)$ \\
\hline Other & 2,340 & $327(14.0)$ & $105(4.5)$ & $21(0.9)$ \\
\hline Total & 24,874 & $6,531(26.3)$ & $2,374(9.5)$ & $637(2.6)$ \\
\hline
\end{tabular}

S/D/W separated, divorced, or widowed.

a $P$ value derived from $\chi^{2}$ tests.

use and cannabis use in pregnancy. Almost $8 \%$ of Indigenous women reported using cannabis during pregnancy compared with 3\% for Caucasian women. All associations were significant at the 0.001 level.

The associations between lifetime use, ever regular use, and pregnancy use of cannabis and birth outcomes are presented in Table 2. Univariate analyses show that as compared with non-cannabis-using women, babies of women who smoked cannabis during pregnancy were, on average, $375 \mathrm{~g}$ lighter, had a lower gestational age, shorter body length, and were more likely to be SGA and to be admitted to the neonatal intensive care unit (NICU). The data also show that ever regular use of cannabis was associated with lower birth weight and birth length, with being SGA, and with admission to NICU. There was no significant association between use of cannabis before or during pregnancy and Apgar score at $5 \mathrm{~min}$.
The potential impact of cannabis use during pregnancy might be confounded by maternal factors that are related to both cannabis use and birth outcomes. These include mother's socioeconomic status, mother's weight, cigarette smoking, and alcohol consumption during pregnancy. In Table 3, the relative impact of cannabis use during pregnancy on negative birth outcomes is adjusted for potential confounders. Unadjusted analysis shows that risk of low birth weight (less than $2,500 \mathrm{~g}$ ) was more than twice (odds ratio $(\mathrm{OR})=2.4$; 95\% confidence interval (CI): 1.9-3.0) for babies of women using cannabis during pregnancy. It is also noted that the odds of overweight (above $4,000 \mathrm{~g}$ ) birth was $70 \%$ less for these women. Adjustment for potential confounders did not materially change the associations. Multivariate analyses also showed that low birth weight ( $\mathrm{OR}=1.7 ; 95 \% \mathrm{CI}: 1.3-2.2)$, preterm birth ( $\mathrm{OR}=1.5 ; 95 \% \mathrm{CI}: 1.1-1.9)$, SGA $(\mathrm{OR}=2.2 ; 95 \%$ CI: $1.8-2.7)$, and admission to the NICU (OR $=2.0 ; 95 \%$ CI: $1.7-2.4)$ were statistically significantly associated with cannabis use during pregnancy.

\section{Sensitivity Analysis}

Of 6,531 women who reported lifetime use of cannabis, 9.7\% $(n=632)$ had used cannabis during pregnancy, and of 2,374 women who were regular cannabis users before pregnancy, $22.3 \%(n=529)$ reported cannabis use in pregnancy. Data in Table 2 indicated that ever regular cannabis use was associated with birth weight, birth length, SGA, and admission to the NICU. In complementary analyses, we examined the effect of lifetime and regular use of cannabis before pregnancy on birth outcomes, excluding those women who had used cannabis during pregnancy. These analyses did not show significant association between lifetime and regular use of cannabis before pregnancy and birth weight, preterm birth, and admission to the NICU (data not shown).

In another series of analyses, we tested the association between cannabis use before and during pregnancy and neonatal outcomes (length of stay in neonatal nursery, neonatal death, and use of mechanical ventilation). Our data did not show any significant association between use of cannabis either before or during pregnancy and these three outcomes.

To estimate the overall impact of cannabis use during pregnancy on negative birth outcomes, we calculated the population-attributable risk. The population-attributable risk uses the OR and prevalence of the exposure to estimate the maximum proportion of negative outcome that could be prevented if the population were free of the exposure (cannabis use in pregnancy). Using ORs from multivariate adjusted analysis (Table 3), the population-attributable risk for low birth weight, preterm birth, and ICU admission were 2.5, 1.5, and 2.7\%, respectively.

\section{DISCUSSION}

This study presents data from a large cohort of women presenting for public antenatal care at a large tertiary maternity hospital in Brisbane, Australia, over a 7-y period. Overall, $2.6 \%$ of women reported currently using cannabis at their 
Table 2. Univariate association of cannabis use with birth outcomes, Mater Mothers' Hospital, 2000-2006

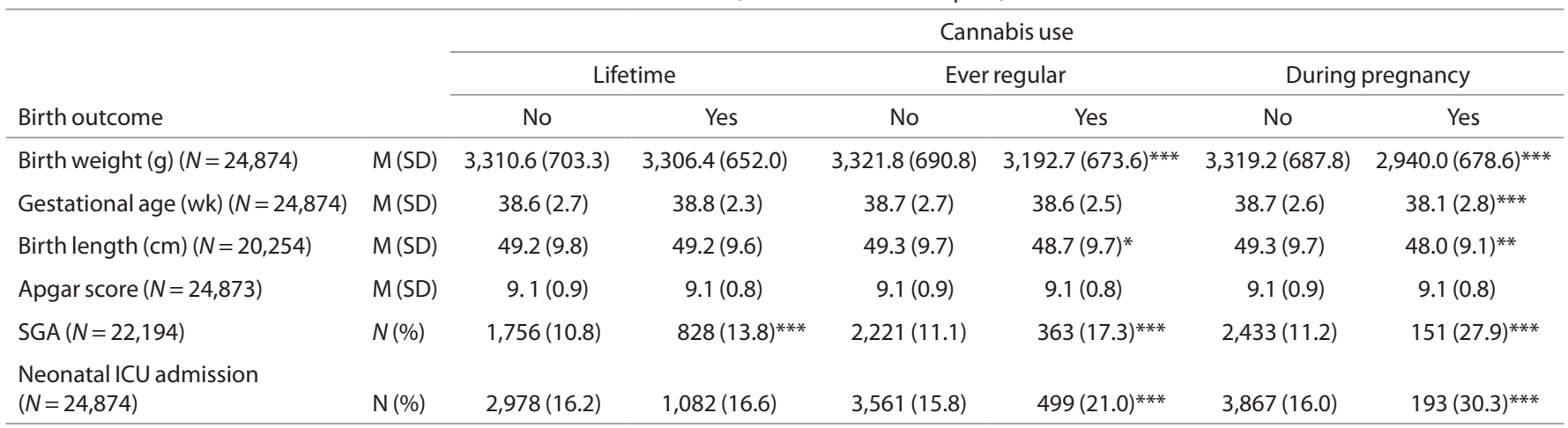

Differences are significant at ${ }^{*} P$ value $<0.05,{ }^{* *} P$ value $<0.01,{ }^{* *} P$ value $<0.001$.

ICU, intensive care unit; $M$, mean; SGA, small for gestational age.

Table 3. Adjusted association of cannabis use during pregnancy with birth outcome, Mater Mothers'Hospital, 2000-2006

\begin{tabular}{|c|c|c|c|}
\hline \multirow[b]{3}{*}{ Birth outcome } & \multicolumn{3}{|c|}{ Cannabis use during pregnancy } \\
\hline & Unadjusted & Adjusted $^{a}$ & Adjusted $^{b}$ \\
\hline & OR $(95 \% \mathrm{Cl})^{\mathrm{c}}$ & OR $(95 \% \mathrm{Cl})^{\mathrm{c}}$ & OR $(95 \% \mathrm{Cl})^{\mathrm{c}}$ \\
\hline \multicolumn{4}{|l|}{ Birth weight } \\
\hline$<2,500 \mathrm{~g}$ & $2.4(2.0-2.9)^{* *}$ & $2.3(1.9-2.9)^{* *}$ & $1.7(1.3-2.2)^{* *}$ \\
\hline $2,500-4,000 \mathrm{~g}$ & Ref & Ref & Ref \\
\hline$>4,000 \mathrm{~g}$ & $0.3(0.2,0.5)^{* *}$ & $0.3(0.2-0.5)^{* *}$ & $0.5(0.3-0.8)^{*}$ \\
\hline \multicolumn{4}{|l|}{ Preterm birth } \\
\hline No & Ref & Ref & Ref \\
\hline Yes & $1.7(1.4-2.1)^{* *}$ & $1.7(1.3-2.1)^{* *}$ & $1.5(1.1-1.9)^{*}$ \\
\hline \multicolumn{4}{|l|}{ SGA } \\
\hline No & Ref & Ref & Ref \\
\hline Yes & $3.1(2.5-3.7)^{* *}$ & $3.1(2.5-3.7)^{* *}$ & $2.2(1.8-2.7)^{* *}$ \\
\hline \multicolumn{4}{|l|}{ NICU admission } \\
\hline No & Ref & Ref & Ref \\
\hline Yes & $2.3(1.9-2.7)^{* * *}$ & $2.3(1.9-2.8)^{* *}$ & $2.0(1.7-2.4)^{* *}$ \\
\hline
\end{tabular}

Different from reference category at ${ }^{*} P$ value $<0.01,{ }^{*} P$ value $<0.001$.

$\mathrm{Cl}$, confidence interval; $\mathrm{NICU}$, neonatal intensive care unit; $\mathrm{OR}$, odds ratio; Ref, reference category; SGA, small for gestational age.

aAdjusted for mother's age, parity, ethnicity, and weight. ${ }^{b}$ Adjusted for mother's age parity, ethnicity, weight, cigarette smoking, alcohol consumption, and use of other illicit drugs during pregnancy. 'No use of cannabis considered reference category.

first antenatal clinic visit. Use of cannabis during pregnancy strongly and significantly predicted negative birth outcomes, including low birth weight, preterm birth, SGA, and admission to the NICU. After controlling for mothers' sociodemographic characteristics, smoking, alcohol consumption, and use of other illicit drugs, these increased levels of poor outcomes remained statistically significant.

The findings of this study are consistent with previous research, which has suggested that smoking cannabis during pregnancy may lead to lower birth weight (6-9), increased rate of premature birth (7), and shorter birth length $(11,12)$. However, our data are inconsistent with findings of studies that have not found a significant association between cannabis use in pregnancy and birth outcomes $(13,14,16,18)$. The existing discrepancy between the findings might be due to the difference in the study design and assessment of cannabis use (e.g., retrospective vs. prospective) (14) or level of adjustment for confounders $(13,16)$. Using a large routinely collected data set, our study suggests a significant effect of in utero exposure to cannabis on the birth outcomes examined independent of sociodemographic background and use of other substances.

Our findings need to be interpreted in the context of a number of limitations. First, the data were derived from self-reports for which there was no objective validation. It can be argued that self-report measurement of substance use during pregnancy is subject to problems of poor instrument design, recall bias, and underreporting (19-22). The issues involved in women's reluctance to reveal their substance use include the illicit nature of some substances, fear of having their children taken into care, fear of legal consequences, shame, guilt, and recall problems (23). An alternative and more reliable measurement is laboratory assessment of the substance in body specimens (e.g., urine, blood, hair). However, studies that rely solely on drug testing are likely to underestimate the prevalence of drug use over a long period of time because drug metabolites are detectable in blood or urine for only a few days after the drugs were last used (24). Studies that have examined validity of selfreported cannabis use have reported good correlation with urinalysis results (4).

Notwithstanding, measurement misclassification could jeopardize the findings of this study if the association between cannabis use and birth outcomes were in the opposite direction. For example, it can happen that those who are not typical cannabis users (e.g., those from a higher socioeconomic background) may be more likely to underreport drug use. If this is the case, underreported cannabis use among women who maintain better birth outcomes may lead to overestimation of the association between cannabis use and adverse birth outcomes. On the other hand, if cannabis use during pregnancy is underreported, regardless of the women's background characteristics, it seems plausible that nondifferential measurement bias might have led to underestimation of the true association. 


\section{Articles $\mid$ Cannabis use and birth outcomes}

Another limitation is that the association observed here might be confounded by some other factors that have not been controlled for. It is possible that those who ever used cannabis have been exposed to different behavioral and environmental factors as compared with those who used cannabis during pregnancy or those who never used it. Although we corrected the associations for a range of potential confounders, there remains a possibility that the observed effects of cannabis use during pregnancy are due to unmeasured or residual confounding. In addition, our data do not include women presenting to private hospitals. This may cause a limitation in the generalizing of the findings to a population of higher socioeconomic status. Finally, although our data do now show a significant relationship between cannabis use in pregnancy and the three neonatal outcomes, lack of access to the long-term follow-up does not allow us to examine the potential effect of cannabis on the health of the participants included in this study. The clinical significance of adverse effects of cannabis use during pregnancy would be more remarkable if children's growth and development could be monitored subsequently.

In general, in this large population of women, the prevalence of cannabis use before pregnancy was high, whereas only a small proportion of women continued using cannabis during pregnancy. The current data have significant implications for public health. One issue of relevance to policy makers is the relationship between self-reported use of cannabis by pregnant women and adverse birth outcomes. If we assume there is a causal relationship between the exposure and outcome, $2.5 \%$ of low birth weight, $1.5 \%$ of preterm delivery, and $2.7 \%$ of admission to the NICU could be prevented if pregnant women did not use cannabis during pregnancy in this tertiary hospital setting.

\section{Conclusions}

The number of women who reported using cannabis in pregnancy was low, but with large numbers of births now concentrated in a small number of maternity units, there is an opportunity to extend services in pregnancy as well as to provide community outreach services for these women after their children are born. New efforts should focus on educating women and health-care providers about the consequences of cannabis use and encouraging women to stop smoking cannabis before becoming pregnant. Further research is needed to examine associations with birth outcomes by the quantity of cannabis used as well as the longterm effect of cannabis use during pregnancy on offspring.

\section{METHODS}

\section{Participants}

This study was undertaken at the MMH, one of the two tertiary maternity hospitals in Brisbane, Australia, that provide specialist obstetric services. The MMH is located on the south side of the Brisbane River and services a population comprising diverse socioeconomic groups. It coexists with Mater Mothers' Private Hospital and shares a campus with Mater Adult (public and private) Hospitals and Mater Children's (public and private) Hospitals. Currently, MMH (public and private hospitals) birth around 10,000 babies a year. Pregnant women attending the $\mathrm{MMH}$ are considered to be from middle and lower income population as compared with those attending the private hospital. This study is based on routinely collected data for 24,874 women attending for public antenatal care at the MMH over the 7-y period 2000-2006. All women who book in to the MMH undergo a "booking-in history." The booking-in histories are generally conducted between 12 and 16 wks gestation. The alcohol and drug questions were part of this booking-in history. Non-English-speaking women were provided with interpreters relevant to their identified language.

\section{Instruments}

In 1999, in an effort to identify women to refer to a new alcohol and drug use antenatal clinic at the $\mathrm{MMH}$, an alcohol and drug use questionnaire was developed and incorporated into the broader hospital booking interview. This questionnaire was compiled with the assistance of midwife-interviewers and was adapted from comprehensive alcohol and drug use assessments that were in common use at the time. All midwives working in the antenatal clinic were instructed on standard drinks measures, specific drugs (and street names) and their effects, recommendations for abstinence in pregnancy, interviewing techniques/barriers to effective communication, how to use the database, and referring women to relevant services.

\section{Outcome Variables}

Information on the outcome of each pregnancy was routinely collected by the midwives and entered into an Obstetric Clinical Reporting System. A research database consisting of de-identified data was accessed by researchers at the Mater Mothers' Research Centre. The outcomes of interest in this study were low birth weight, preterm delivery, birth length, Apgar score in the fifth minute, SGA, and admission to the NICU. The baby's birth weight was measured to the nearest $1 \mathrm{~g}$. Low birth weight was defined as less than 2,500 g. Gestation (number of completed weeks) was based on an early ultrasound scan or, if not available, on the date of the last menstrual period and/or on clinical assessment. Preterm birth was defined as a birth that occurred before 37 completed weeks of gestation. Birth weight standards developed by Pain et al. (25) were used to define a population of infants who were SGA. According to this model, infants with a birth weight less than the tenth percentile adjusted for gender, gestational age, ultrasound validated gestation, mother's age, BMI, height, parity, and ethnicity are defined as SGA.

\section{Independent Variables}

MMH public booking interviews were usually conducted early in the women's second trimester of pregnancy. Women were questioned about whether they had ever used and ever regularly used a range of illicit drugs, including cannabis, amphetamines, ecstasy, and heroin. Any self-reported past use (ever use) and any regular use (ever regular use) of these substances was recorded. Women who reported ever use of illicit drugs were asked whether they were currently using these drugs. Women who reported current use or having recently quit using substances were offered antenatal care through the hospital's specialist alcohol and drug service. Women were also asked about use of alcohol, tobacco, and prescribed medications, including benzodiazepines, methadone, and buprenorphine (buprenorphine was introduced as an opioid replacement therapy in Australia in 2000-2001 and was added to the database later). Responses regarding routinely collected obstetric data were entered in the MMH electronic Clinical Reporting System by the midwife undertaking the interview. The same questionnaire was used throughout the study period (2000-2006) with only minor changes (including measurement of tobacco use and addition of buprenorphine to the questionnaire).

\section{Other Variables}

Information on women's sociodemographic information, weight, smoking, and alcohol consumption were also collected at their visit to the antenatal clinic. Maternal age (number of completed years), highest level of education (tertiary education, completed high school, and incomplete high school), ethnicity, and parity were assessed at the first antenatal booking visit. According to their age, women were divided into three categories (below 20 y, 20-35 y, and older than 35 y) for univariate analysis only. Ethnicity was ascertained by the midwife from the women's self-reporting at the antenatal booking visit and, for the analysis, was classified into four groups: Caucasian, Indigenous, Asian, and Other. The term "Indigenous" was used to refer to women 
who were recorded as of Aboriginal and/or Torres Strait Islander descent. Parity was defined as the number of previous births of $20 \mathrm{wks}$ or more gestation, which was further categorized into four groups $(0,1,2$, and $3+)$ for analysis purposes.

\section{Statistical Analyses}

A de-identified data set was extracted from the Clinical Reporting System for all women birthing at the $\mathrm{MMH}$ over the study period. A total of 24,874 women who gave birth to live babies over this period were included in the study. First, we used descriptive analyses to compare use of cannabis before and during pregnancy according to the women's sociodemographic characteristics. A series of frequency tables, $\chi^{2}$ tests, and Student's $t$ tests (where the outcome variable was continuous) were used to examine the association of cannabis use before and during pregnancy with birth outcomes. We then used logistic regression to calculate the OR and associated 95\% CI relating birth outcomes to the use of cannabis before and during pregnancy. We adjusted associations for mother's age, level of education, marital status, ethnicity, parity, weight, cigarette smoking, alcohol consumption, use of other illicit drugs (heroin, amphetamines, ecstasy, and hallucinogens), and use of prescription drugs (including methadone).

We also conducted a series of additional analyses to examine the association between cannabis use before and during pregnancy and three neonatal outcomes (including length of stay in neonatal nursery, neonatal death, and use of mechanical ventilation). Finally, we used ORs from multivariate logistic regression to estimate the population-attributable risk for low birth weight, preterm birth, and NICU admission due to cannabis use during pregnancy. Analyses were carried out using Stata/SE 10.0 (Stata, College Station, TX), and a $P$ value less than 0.05 was considered significant.

\section{Ethics Committee Approval}

In accordance with the National Health and Medical Research Council criteria, this study was deemed a quality-assurance activity exempt from the need for a full ethics review by the Mater Health Services Human Research Ethics Committee. The Mater Health Services Human Research Ethics Committee reviewed this study and assessed it to be a unique quality-assurance project, part of which involved the collection and analysis of retrospective data.

\section{STATEMENT OF FINANCIAL SUPPORT}

Financial support for this study was provided by the University of Queensland, Australia.

\section{REFERENCES}

1. Richardson R, Bolisetty S, Ingall C. The profile of substance-using pregnant mothers and their newborns at a regional rural hospital in New South Wales. Aust N Z J Obstet Gynaecol 2001;41:415-9.

2. Substance Abuse and Mental Health Services Administration. Results From the 2007 National Survey on Drug Use and Health: National Findings (NSDUH Series H-34, DHHS Publication No. SMA 08-4343). Rockville, MD: Department of Health and Human Services, 2008.

3. Australian Institute of Health and Welfare. National Drug Strategy Household Survey: First Results. Canberra: AIHW2008, 2007.

4. Zuckerman B, Frank DA, Hingson R, et al. Effects of maternal marijuana and cocaine use on fetal growth. N Engl J Med 1989;320:762-8.
5. Bell GL, Lau K. Perinatal and neonatal issues of substance abuse. Pediatr Clin North Am 1995;42:261-81.

6. Hingson R, Alpert JJ, Day N, et al. Effects of maternal drinking and marijuana use on fetal growth and development. Pediatrics 1982;70:539-46.

7. Hatch EE, Bracken MB. Effect of marijuana use in pregnancy on fetal growth. Am J Epidemiol 1986;124:986-93.

8. Sherwood RA, Keating J, Kavvadia V, Greenough A, Peters TJ. Substance misuse in early pregnancy and relationship to fetal outcome. Eur J Pediatr 1999;158:488-92.

9. Kline J, Stein Z, Hutzler M. Cigarettes, alcohol and marijuana: varying associations with birthweight. Int J Epidemiol 1987;16:44-51.

10. Gibson GT, Baghurst PA, Colley DP. Maternal alcohol, tobacco and cannabis consumption and the outcome of pregnancy. Aust N Z J Obstet Gynaecol 1983;23:15-9.

11. Tennes K, Avitable N, Blackard C, et al. Marijuana: prenatal and postnatal exposure in human. In: Pinkert TM, ed. NIDA Research Monograph 59. Rockville, MD: National Institute on Drug Abuse, 1985:48-62.

12. Day N, Sambamoorthi U, Taylor P, et al. Prenatal marijuana use and neonatal outcome. Neurotoxicol Teratol 1991;13:329-34.

13. Lang JM, Lieberman E, Cohen A. A comparison of risk factors for preterm labor and term small-for-gestational-age birth. Epidemiology 1996;7:369-76.

14. Linn S, Schoenbaum SC, Monson RR, Rosner R, Stubblefield PC, Ryan KJ. The association of marijuana use with outcome of pregnancy. Am J Public Health 1983;73:1161-4.

15. Fergusson DM, Horwood LJ, Northstone K. Maternal use of cannabis and pregnancy outcome. BJOG 2002;109:21-7.

16. Shiono PH, Klebanoff MA, Nugent RP, et al. The impact of cocaine and marijuana use on low birth weight and preterm birth: a multicenter study. Am J Obstet Gynecol 1995;172(1 Pt 1):19-27.

17. Fried PA, O'Connell CM. A comparison of the effects of prenatal exposure to tobacco, alcohol, cannabis and caffeine on birth size and subsequent growth. Neurotoxicol Teratol 1987;9:79-85.

18. Witter FR, Niebyl JR. Marijuana use in pregnancy and pregnancy outcome. Am J Perinatol 1990;7:36-8.

19. Day NL, Richardson GA. Prenatal marijuana use: epidemiology, methodologic issues, and infant outcome. Clin Perinatol 1991;18:77-91.

20. Pegues DA, Engelgau MM, Woernle CH. Prevalence of illicit drugs detected in the urine of women of childbearing age in Alabama public health clinics. Public Health Rep 1994;109:530-8.

21. Lester BM, ElSohly M, Wright LL, et al. The Maternal Lifestyle Study: drug use by meconium toxicology and maternal self-report. Pediatrics 2001;107:309-17.

22. Hingson R, Zuckerman B, Amaro H, et al. Maternal marijuana use and neonatal outcome: uncertainty posed by self-reports. Am J Public Health 1986;76:667-9.

23. Koren G, Chan D, Klein J, Karaskov T. Estimation of fetal exposure to drugs of abuse, environmental tobacco smoke, and ethanol. Ther Drug Monit 2002;24:23-5.

24. Chiang C, Hawks R. Implications of drug levels in body fluids: basic concepts. NIDA Research Monograph Series No 79. Rockville, MD: National Institute on Drug Abuse, 1986.

25. Pain S, Chang AM, Flenady V, Chan FY. Customised birthweight: coefficients for an Australian population and validation of the model. Aust N Z J Obstet Gynaecol 2006;46:388-94. 